\title{
ALTERAÇÕES NEUROMUSCULARES EM IDOSOS COM DOENÇA DE PARKINSON
}

\author{
Ana Paula Janner Zanardi ${ }^{1}$ \\ Elren Passos Monteiro² \\ Valéria Feijó Martins ${ }^{3}$ \\ Leonardo Alexandre Peyré-Tartaruga ${ }^{4}$
}

resumo

Ao passo que distúrbios de movimento são reconhecidos na doença de Parkinson (DP), os fatores neurais relacionados à doença integrados ao envelhecimento são menos compreendidos. O objetivo desta revisão narrativa foi analisar os principais mecanismos neurais envolvidos na redução da força em idosos com DP e verificar o papel da caminhada nórdica (CN) na estrutura e função muscular em pessoas com DP. Uma pesquisa bibliográfica foi realizada nas bases Lilacs, PubMed e SciELO com os descritores: aging, Parkinson

1 Fisioterapeuta, estudante de mestrado pelo Programa de Pós-Graduação em Ciências do Movimento Humano da Universidade Federal do Rio Grande do Sul (UFRGS). E-mail: anapjzanardi@gmail.com.

2 Mestre. Doutoranda pelo Programa de Pós-Graduação em Ciências da Saúde da Universidade Federal de Ciências da Saúde de Porto Alegre (UFCSPA). E-mail: elren.monteiro@gmail.com.

3 Mestre. Doutoranda no Programa de Pós-Graduação em Ciências do Movimento Humano da Universidade Federal do Rio Grande do Sul (UFRGS). E-mail: valeriafeijo4@gmail.com.

4 Professor Doutor da Escola de Educação Física, Fisioterapia e Dança da Universidade Federal do Rio Grande do Sul (UFRGS). E-mail: leonardo.tartaruga@ufrgs.br. 
disease, muscle, neuromuscular disease e nervous system. Limitou-se a artigos em inglês e português de 1997 a 2018. Foram encontrados 573 artigos, foram selecionados 53 artigos, foram usados 15, e 8 foram incluídos através de buscas manuais. A fraqueza muscular em idosos com DP dá-se pelas alterações centrais da DP associadas às alterações neurais provenientes do envelhecimento. A CN e dança podem auxiliar na melhora intrínseca de pessoas com DP. No entanto, a literatura é escassa quando se trata deste assunto. Com isso, são necessários estudos que abordem análises cinéticas e cinemáticas após a prática de CN e dança em pessoas com DP.

palavras-chave

Envelhecimento. Doenças Neurodegenerativas. Doenças Neuromusculares. Sistema Nervoso. Força Muscular.

\section{Introdução}

O envelhecimento é complexo e multifatorial, e a senescência celular indica a finitude na capacidade de muitas células de dividirem-se, renovarem-se e regenerarem-se, ou seja, o envelhecimento é um processo irreversível (LABAT-ROBERT; ROBERT, 2015). Segundo a Organização Mundial da Saúde (OMS), em países em desenvolvimento a população idosa é definida com idade igual ou acima dos 60 anos, enquanto que em países desenvolvidos a faixa etária para ser considerado idoso é de 65 anos ou mais (WHO, 2009). Dados epidemiológicos revelam que a população mundial está envelhecendo, entre 1999 e 2009 houve um aumento de 11,3\% na população idosa no Brasil, e até 2025 estima-se que a população idosa brasileira seja a sexta maior do mundo (IBGE, 2009).

Com o processo do envelhecimento, as doenças neurodegenerativas tornam-se frequentes, sendo o Alzheimer a mais prevalente, enquanto que a doença de Parkinson (DP) é a segunda mais comum a afetar pessoas com 60 anos ou mais (FLORIANO et al., 2015). Há indícios que até 2050 os casos de DP irão duplicar no mundo (FLORIANO et al., 2015). Estudos apresentam que o envelhecimento é o principal fator de risco para a DP (RODRIGUEZ et al., 2015; COLLIER; KANAAN; KORDOWER, 2017). Ou seja, o desenvolvimento da doença pode ser dependente da idade, sendo a incidência de aproximadamente $0,5 \%$ a $1 \%$ para pessoas entre 65 e 69 anos e 1 a 3\% naquelas com 80 anos ou mais. Estima-se que a prevalência da DP aumentará em decorrência 
do aumento da população idosa (BRIENESSE; MICHAEL, 2013; RODRIGUEZ et al., 2015; COLLIER; KANAAN; KORDOWER, 2017).

A DP é uma desorganização crônica, progressiva e polissintomática do sistema nervoso central, associada a disfunções do movimento (ALLEN et al., 2009), que pode provocar alterações cognitivas, comportamentais e de humor, além da demência que, ocorre em $25 \%$ dos indivíduos afetados. Estas alterações iniciam de forma gradual e lenta e tendem a agravar com o tempo. Os sintomas motores são os principais na DP, nomeadamente, a rigidez muscular, bradicinesia definida pela lentidão na execução do movimento, tremores de repouso, falta de coordenação motora, instabilidade postural, disfunção da marcha e redução de força muscular (LAMOTTE et al., 2015; MONTEIRO et al., 2017; COLLIER; KANAAN; KORDOWER, 2017). As alterações periféricas em idosos com DP, como a fraqueza muscular, que promovem aumento de quedas e dependência nas atividades de vida viária, podem ser causadas por alterações da comunicação ao nível central (DESCHENES, 2004; PÍCOLI; FIGUEIREDO; PATRIZZI, 2011; WU et al., 2016). Embora se saiba que o envelhecimento acarrete fraqueza muscular, as reduções adicionais da força e da massa muscular devido à DP não estão claras.

Estratégias de intervenção desenvolvendo os sistemas múltiplos de memória, inclusive com exercícios cognitivos e motores concomitantes (também denominados de dupla-tarefa) vêm demonstrando resultados promissores em pessoas com DP (POLDRACK; PACKARD, 2003; WILD et al., 2013). Entre estas intervenções, a caminhada nórdica $(\mathrm{CN})$ apresenta uma integração cortical mais complexa do que a caminhada livre, com padrões de coordenação e ativação muscular mais intensa devido à geração de trabalho mecânico para propulsão dos membros superiores (PELLEGRINI et al., 2015; PELLEGRINI et al., 2017). Outra atividade que vem sendo estudada é a prática de dança, que por apresentar movimentos rítmicos e coordenados pode auxiliar na manutenção de amplitude de movimento, coordenação e controle motor (SHARP; HEWITT, 2014; SHANAHAN, et al., 2015).

A compreensão global das alterações neurais que afetam o sistema muscular em idosos com DP podem auxiliar no melhor entendimento dos mecanismos adaptativos neurais provenientes da DP e podem auxiliar na prescrição de exercícios. Diante do exposto, o presente estudo tem por objetivo revisar de modo narrativo as principais alterações neurais que afetam o sistema muscular em idosos com DP, além de investigar como a $\mathrm{CN}$ e a dança podem atuar nas adaptações neuromusculares de idosos com DP a fim de auxiliar na mobilidade destes indivíduos. 
O presente estudo é uma revisão da literatura, do tipo narrativa, que abordará o tema: alterações neuromusculares em idosos com DP. Fez-se uma pesquisa bibliográfica nos bancos de dados dos sistemas Lilacs, PubMed e SciELO com os seguintes descritores em inglês: aging, Parkinson disease, muscle, neuromuscular disease e nervous system. A pesquisa online dos artigos limitou-se aos idiomas inglês e português publicados no período de 1997 a 2018.

Como critério de inclusão, os artigos deveriam apresentar no título e/ou resumo as palavras chaves envelhecimento, DP, doenças neuromusculares e sistema nervoso. Foram excluídos artigos que não apresentaram os termos descritos acima e que não abordaram a temática. Primeiramente foram encontrados 573 artigos, após exclusão das duplicatas e leitura manual dos títulos e resumos, foram selecionados 53 artigos para leitura completa. Destes, foram usados 15 artigos, e 8 foram incluídos através de buscas manuais. Por se tratar de uma revisão narrativa, não houve a avaliação da qualidade dos estudos.

A partir desta busca a revisão foi estruturada da seguinte forma: 1) Unidade motora, junção neuromuscular e envelhecimento neural; 2) Características da DP; 3) Adaptações neuromusculares em idosos com DP; 4) CN nas adaptações neuromusculares de idosos com DP; 5) Dança nas adaptações neuromusculares de idosos com DP.

\section{Resultados}

O presente estudo teve por objetivo revisar quais as principais alterações neurais que afetam o sistema muscular em idosos com DP. Os artigos revisados mostram que na DP ocorre morte dos neurônios dopaminérgicos da substância negra, o que irá desencadear a diminuição das respostas eferentes das vias extrapiramidais do sistema nervoso central. Estas alterações, associadas às alterações piramidais do processo de envelhecimento, irão causar redução nas respostas dos motoneurônios, que implica em mudanças na comunicação neural e muscular. Consequentemente, haverá redução do tempo de ativação muscular, do recrutamento de unidades motoras e da força muscular. A eficácia de exercícios aeróbicos aos níveis centrais e periféricos são conhecidos, a $\mathrm{CN}$, dentre os exercícios aeróbicos, mostrou-se um método eficaz nas adaptações neuromusculares de idosos com DP, proporcionando melhoras funcionais aos seus praticantes. As mudanças de ritmos e o estímulo auditivo presente na dança podem proporcionar adaptações neuromusculares em idosos com DP. Os capítulos a seguir são destinados a maior compreensão e aprofundamento dos achados. 
A produção de força e movimento é desencadeada por um grupo de fibras musculares que são inervadas pelo mesmo neurônio motor inferior (ou motoneurônio $\alpha$ ), denominada de unidade motora. As unidades motoras são homogêneas, um motoneurônio inerva sempre o mesmo tipo de fibra muscular e quanto maior o número de unidades motoras ativadas, maior será a quantidade de força produzida pelo músculo (MANINI; HONG; CLARK, 2013). Em condições normais, quando o neurônio motor transmite um impulso, o músculo se contrai. As propriedades estruturais, como por exemplo, o tamanho do motoneurônio, irá determinar a ordem/sequência da ativação muscular (MANINI; HONG; CLARK, 2013).

No envelhecimento é possível evidenciar a progressiva perda neural, que acontece inicialmente no cérebro, na região dos giros pré-frontais do córtex e é dissipada para todo o sistema nervoso periférico. Esta diminuição de neurônios altera a comunicação intraneuronal central, que traz consequências negativas ao nível periférico, como a fraqueza muscular (MANINI; HONG; CLARK, 2013; TUDORAŞCU, 2014; KWON; YOON, 2017).

Estas alterações centrais e periféricas causam mudanças na arquitetura, morfologia e na propriedade funcional da musculatura dos idosos (HUNTER; PEREIRA; KEENAN, 2016). As degenerações neuronais centrais provocam a diminuição na velocidade do disparo do potencial de ação dos axônios para as fibras musculares (TUDORAŞCU, 2014; HEPPLE; RICE, 2016) e esta diminuição da velocidade acaba provocando degeneração muscular. Consequentemente, há diminuição da propagação do estímulo, principalmente nas fibras musculares tipo II, promovendo a diminuição no tamanho do músculo e da força muscular, tornando os indivíduos fragilizados e susceptíveis a lesões (HEPPLE; RICE, 2016; KWON; YOON, 2017).

\subsection{Características da DP}

A DP é uma desorganização crônica, progressiva e polissintomática do sistema nervoso central, caracterizada pelo acúmulo anormal da proteína pré-sináptica alfa-sinucleína nos corpos de Lewy que se acumulam em regiões intraneuronais e intracitoplasmáticas. Este agregado anormal desta proteína, também é conhecido como sinucleínopatia (PERFEITO; REGO, 2012; SCHNEIDER; ALCALAY, 2017). 
A literatura apresenta que, apesar de eminentemente pesquisada, as causas da DP não estão esclarecidas. Há evidências de que fatores ambientais e genéticos estão envolvidos no desenvolvimento da doença e além disso, o envelhecimento é considerado um fator de risco para o surgimento da DP (FLORIANO et al., 2015; RODRIGUEZ et al., 2015). Antes dos sintomas motores se manifestarem, pelo menos $50 \%$ dos neurônios dopaminérgicos da substância negra apresentam comprometimento e morte neuronal, o que afeta o desenvolvimento e a manutenção da força das unidades motoras em indivíduos afetados pela DP (BARGHI; GLADDEN, 2013; HAMMOND et al., 2017).

Estas desordens determinam à diminuição da produção do neurotransmissor dopamina que atua principalmente no controle dos movimentos. A diminuição de dopamina ocorre na parte compacta da substância negra e a carência deste neurotransmissor reflete em alterações na região do planejamento e sequenciamento dos movimentos, denominadas de núcleos da base (BARGHI; GLADDEN, 2013; HAMMOND et al., 2017; PETERSON; HORAK, 2016).

Os núcleos da base possuem duas vias, a via direta e a indireta. Na DP tem-se a diminuição da dopamina na via direta o que reduz a atividade nas áreas motoras do córtex cerebral, promovendo diretamente alterações na via extrapiramidal. Quando esta via é afetada, o indivíduo possui movimentos irregulares e sem coordenação, além de dificuldade para iniciar e controlar os movimentos (PETERSON; HORAK, 2016).

As alterações nos núcleos da base que ocorrem pela DP são associadas à redução da excitabilidade e plasticidade cortical provenientes do envelhecimento (PETERSON; HORAK, 2016). A bradicinesia, alterações posturais, diminuição do controle motor e consequente fraqueza muscular presentes na DP, são decorrentes da degeneração da substância negra compacta que resulta na inibição do globo pálido externo, interno e tálamo. A cinemática de idosos com DP é afetada pela combinação de fraqueza muscular e menor controle motor, pois a menor velocidade da marcha e menor comprimento da passada possuem correlação com alterações no sistema dopaminérgico e colinérgico. A bradicinesia relaciona-se principalmente às alterações de vias dopaminérgicas, enquanto que movimentos involuntários, alterações posturais e rigidez são relacionados com alterações colinérgicas (PETERSON; HORAK, 2016).

Estas alterações vão gerar respostas motoras eferentes danificadas, que resultam no recrutamento insuficiente de unidades motoras responsáveis pela produção de força para iniciar o movimento. Consequentemente, ocorre a redução da força principalmente pelo fator do envelhecimento e diminuição na velocidade do movimento pelas características advindas da DP (RODRIGUEZ et al., 2015; WU et al., 2016; HAMMOND et al., 2017). 

número (hipoplasia) e do tamanho (hipotrofia) das fibras musculares, principalmente nas fibras musculares do tipo II tanto em idosos saudáveis quanto em idosos com DP (JANKELOWITZ; BURKE,2012; WU et al.,2016).

No processo de envelhecimento o número de células nervosas reduz aproximadamente $7 \%$ por década. Desta forma, em idosos com DP haverá o acúmulo das perdas neurais provocadas pelo envelhecimento e as perdas decorrentes da doença (COLLIER; KANAAN; KORDOWER, 2017).

A literatura apresenta que mais que um sintoma secundário, a fraqueza muscular é o primeiro sintoma presente em pessoas com DP, explicada pelo fato que na DP os gânglios da base são afetados, com isso as informações de chegada ao córtex motor estão prejudicadas, provocando menor ativação de neurônios motores centrais e periféricos, gerando fraqueza muscular (GOODWIN et al., 2008). Idosos com DP e sem DP diferem entre si em relação ao tempo para atingir a força muscular máxima (taxa de produção de força muscular). Idosos que apresentam a doença despendem cerca de três a quatro segundos para atingir o pico máximo de força de quadríceps enquanto idosos saudáveis realizam a contração aproximadamente em um segundo, sendo, portanto diferente o tempo de ativação muscular, recrutamento de unidades motoras e força muscular (CATALÁ; WOITALLA; ARAMPATZIS, 2013; HAMMOND et al., 2017).

Estudos mostraram que indivíduos com DP são $45 \%$ mais fracos, $43 \%$ menos potentes e apresentam menor velocidade na realização dos movimentos do que pessoas não acometidas, associando-se assim a fraqueza muscular à bradicinesia, bem como menor velocidade e amplitude de sinal eletromiográfico para membros inferiores. Enquanto que a massa corporal, o perímetro de membros inferiores e a área de secção transversa não sofrem influência da DP (HAMMOND et al., 2017).

Como visto anteriormente, a taxa de produção de força é significativamente menor quando estimulada voluntariamente na DP, porém quando estimuladas involuntariamente reduções não foram observadas (HAMMOND et al., 2017). Esse comportamento pode ser explicado pelos distúrbios na velocidade da taxa de desenvolvimento de força, que são de natureza central, provavelmente ligado à excitabilidade cortical. Ao correlacionar resultados da ressonância magnética (RM) com sintomas apresentados por idosos independentes que possuíam DP, percebeu-se que 43\% apresentaram bradicinesia, tremor ou distúrbio da marcha. Além disso, foi verificada correlação de atrofia da área 
sensório motora primária e médio temporal do córtex com a bradicinesia e alteração da marcha em idosos com DP (HUNTER et al., 2016).

Ao observar a ativação muscular e as características biomecânicas de idosos com e sem DP, foi observado que a velocidade média de caminhada foi menor no grupo DP $\left(3,24 \pm 1,22 \mathrm{~km} \mathrm{~h}^{-1}\right)$ quando comparado às pessoas sem acometimento $\left(3,74 \pm 0,94 \mathrm{~km} \mathrm{~h}^{-1}\right)$. Além disso, os picos de ativação muscular de membros inferiores aconteceram mais tarde no ciclo da marcha no grupo DP do que nas pessoas sem acometimento. Por exemplo, o primeiro pico de ativação muscular do grupo DP ocorreu aos 14,74\% da marcha enquanto que controle ocorreu aos 2,24\% (RODRIGUEZ et al., 2013). Os indivíduos com DP apresentam padrões de ativação muscular mais baixo que indivíduos sem DP, principalmente nos músculos gastrocnêmico medial, sóleo e tibial anterior. A baixa ativação destes músculos pode ser uma estratégia compensatória advinda das alterações no controle da atividade cortical. Além da deterioração de força por fatores neurais diretos, ou seja, adversidades de controle como o tremor de repouso e o congelamento dos movimentos (BROWN; CORCOS; ROTHWELL, 1997), respondem também como mecanismos adicionais à resposta de fraqueza muscular em idosos com DP.

As disfunções centrais provenientes da DP ocorrem nos gânglios da base, que possuem papel importante no controle da locomoção. Por sua vez, a degradação dos neurônios colinérgicos e dopaminérgicos na região dos gânglios da base provocam deficiências nas vias eferentes em idosos com DP. Estas alterações centrais refletem em sintomas periféricos como, anormalidades posturais, tremores e bradicinesia que prejudicam o desempenho da marcha, resultando em menor comprimento do passo, menor velocidade de caminhada, menor força do músculo quadríceps (HUANG; FUENTE-FERNÁNDEZ; STOESSL, 2003). Além disso, há evidências de menor ativação dos músculos multifidus e oblíquos externos, que são responsáveis pela manutenção postural estática e dinâmica (COLE; NAUGHTON; SILBURN, 2017). Alterações estas que influenciam diretamente na independência destes indivíduos.

Com isso, a literatura apresenta que tratamentos farmacológicos auxiliam na melhora do controle do movimento. No entanto, exercícios aeróbicos mostram-se eficientes no controle motor, atividades funcionais e na melhora de força muscular, ocorrendo melhoras centrais e periféricas (SHU et al., 2014). 

A síntese de dopamina é estimulada pelas células dopaminérgicas durante a prática de exercícios aeróbicos, minimizando os sintomas provenientes da DP, além de serem protetores ao surgimento de DP, pela liberação de fatores neurotróficos (GOODWIN et al., 2008). Segundo Fox et al. (2006), a prática de exercícios intensos, complexos e prazerosos, maximizam a plasticidade e a adaptação neural, aumentam a produção de dopamina e promovem maior aprendizado motor. Estudos demonstram que a proliferação neural e a liberação de fatores neurotróficos consequentes da prática de exercícios aeróbicos, promovem maior oxigenação cerebral, que auxiliam em benefícios protetores no surgimento de DP (GOODWIN et al., 2008; MURRAY et al., 2014).

O treinamento da caminhada é uma forma simples, segura e eficaz de exercício físico. Revisões sistemáticas recentes vêm demonstrando o potencial da CN como estratégia de intervenção mais eficiente quando comparada com a caminhada livre sem o uso dos bastões (EBERSBACH et al., 2010; TSCHENTSCHER; NIEDERSEER; NIEBAUER, 2013; MONTEIRO et al., 2017).

A CN é uma atividade desafiadora e, através do uso de dois bastões, indivíduos realizem a marcha com movimentos amplos de membros superiores e impulsão do bastão no solo em determinadas etapas do ciclo de marcha (ARCILA et al., 2017). O uso dos bastões proporcionam maior ativação muscular dos membros superiores pelo aumento da amplitude de movimento, principalmente de ombro, e pela impulsão gerada em cada ciclo da passada (PELLEGRINI et al., 2015; PELLEGRINI et al., 2017).

Por ser uma técnica segura, desafiadora, com oscilações de intensidade, que promove convívio social e que exige principalmente concentração, a CN está sendo aplicada em diferentes populações, como por exemplo, pessoas com doença arterial coronariana, insuficiência cardíaca, hipertensão arterial, hiperlipidemia, doença arterial periférica, obesidade, diabetes mellitus tipo II, doença pulmonar obstrutiva crônica e pessoas com DP (SKÓRKOWSKA-TELICHOWSKA et al., 2016).

As alterações neuromusculares presentes na DP podem ser minimizadas com a prática de exercícios físicos regulares, dentre eles, a $\mathrm{CN}$ tem ganhado destaque na literatura (VAN EIJKEREN et al., 2008; LEE; PARK, 2015; MONTEIRO et al., 2017; GOUGEON; ZHOU; NANTEL, 2017). Pessoas com DP apresentam principalmente diminuição de força muscular de gastrocnêmio medial e músculos posturais, como o latíssimo do dorso, refletindo no aumento da rigidez de tronco e nas alterações posturais da população. Na prática da $\mathrm{CN}$ 
pessoas com DP são beneficiadas principalmente pela gama de músculos que são ativados durante a atividade, dentre eles os músculos predominantemente ativos são gastrocnêmio medial, semitendíneo, bíceps femoral, glúteo médio, trapézio superior, latíssimo do dorso, deltóide porção anterior e posterior, tríceps e bíceps braquial (PELLEGRINI et al., 2015; PELLEGRINI et al., 2018).

Estudos como o de Van Eijkeren et al. (2008), Lee e Park (2015), Monteiro et al. (2017) e Franzoni et al. (2018) demonstram que pessoas com DP podem ser beneficiadas ao praticarem a $\mathrm{CN}$, havendo evidências na melhora do equilíbrio, parâmetros espaços temporais, mobilidade articular e melhora da ativação muscular de membros inferiores e superiores. Deste modo, a CN proporciona a combinação de diferentes habilidades, gerando estímulos musculares que podem beneficiar de forma intrínseca e extrínseca idosos com DP, refletindo em melhoras psicológicas, cognitivas e principalmente motoras (VAN EIJKEREN et al., 2008).

As alterações intrínsecas presentes em pessoas com DP refletem na exacerbação dos sintomas da doença, evidências observacionais e longitudinais demonstrem melhoras na mobilidade funcional e aspectos psicossociais com a CN (MONTEIRO et al., 2017). No entanto, faltam estudos que testem a função muscular, o volume muscular e principalmente a cinemática de atividades funcionais após o treinamento de CN em pessoas com DP.

\subsection{Dança nas adaptações neuromusculares de idosos com DP}

O estímulo auditivo advindo da música gera estímulos benéficos aos neurônios motores. Dados eletromiográficos apresentam que a ritmicidade proporcionada pelo som acarreta em contração muscular mais sincronizada e rítmica. Com isso, em populações especiais atividades rítmicas como a dança podem proporcionar melhoras no controle motor e consequentemente na cinemática dos movimentos. A literatura apresenta que terapias que estimulam o ritmo auxiliam na ativação dos músculos gastrocnêmio e tibial anterior durante a marcha (THAUT et al., 1999).

Com isso, a dança surge como potencial intervenção para pessoas com DP, pois o estimulo auditivo associado com as mudanças de direções podem proporcionar melhoras neuromusculares e biomecânicas que irão refletir na qualidade de vida dos seus praticantes (SHARP; HEWITT, 2014; DELABARY et al., 2018). Por ser uma atividade que apresenta ritmo e sincronicidade exige 
atenção, amplitude de movimento, coordenação motora e controle motor dos seus praticantes (SHARP; HEWITT, 2014; SHANAHAN et al., 2015).

O estudo de Allen et al. (2017) apresentou que idosos com DP após três semanas de intervenção de dança de salão apresentaram menor coativação muscular durante a caminhada. Pessoas com DP possuem fraqueza associada a rigidez muscular, com isso, um menor recrutamento muscular após a intervenção pode estar associada a diminuição da rigidez presente nestes indivíduos, além disso foi possível observar relação entre a diminuição do número de fibras atividades e o aumento da coativação muscular (MONTEIRO et al., 2017).

A prática regular de dança proporciona benefícios sociais e motores em pessoas com DP (MCGILL; HOUSTON; LEE, 2014; ALLEN et al., 2017; DELABARY et al., 2018). No entanto, é possível observar que as evidências biomecânicas tanto intrínsecas quanto extrínsecas são escassas quando se trata da dança e idosos com DP, sendo necessário mais estudos que investiguem o efeito da intervenção da dança em melhoras ou manutenção da função muscular e cinemática em pessoas com DP.

\section{Conclusão}

Com a revisão apresentada, foi possível observar que a fraqueza muscular em idosos com DP, ocorre em função das alterações centrais decorrentes da doença associadas às modificações neurais provenientes do envelhecimento. Com isso, a musculatura de idosos com DP tem diferentes padrões de ativação quando comparados a idosos sem DP além de possuir maior tempo de ativação muscular. Deste modo, há diminuição da capacidade de comunicação neural com o músculo, comprometendo a estrutura muscular o que pode interferir na redução adicional na força muscular com implicações importantes nas atividades da vida diária. Atividades como a $\mathrm{CN}$ e dança podem auxiliar na melhora intrínseca de pessoas com DP que consequentemente podem auxiliar em melhoras cinemáticas e funcionais de idosos com DP. A literatura é escassa quando se trata deste assunto. Com isso, são necessários estudos que abordem análises cinéticas e cinemáticas após a prática de $\mathrm{CN}$ e dança em pessoas com DP. 
abstract

While the movement disorders are well determined in Parkinson's disease (PD), these factors integrated to aging process are poorly known. The aim of this narrative review was to analyze the main neural mechanisms involved in the reduction of strength in the elderly with PD. A bibliographic search was performed in the databases Lilacs, PubMed, and SciELO with the descriptors: aging, Parkinson disease, muscle, neuromuscular disease and nervous system. It was limited to articles in English and Portuguese languages from 1997 to 2018. We found 573 articles and we selected 53 articles. We choose 15, and 8 were included through manual searches. Muscle weakness in the elderly with PD is due to central changes associated with neural/peripheral changes from aging. $\mathrm{CN}$ and dance programs may enhance these alterations in people with PD. However, direct evidences on central and peripheral nervous system are scarce. Therefore, effector patterns of the central nervous system may be better evaluated throughout kinetic and kinematic analyzes after $\mathrm{CN}$ and dance training in people with PD

keywords

Aging. Neurodegenerative Diseases. Neuromuscular Diseases. Nervous System. Muscle Strength.

referências

ALLEN, Jessica et al. Increased neuromuscular consistency in gait and balance after partnered, dance-based rehabilitation in Parkinson's disease. Journal of Neurophysiology, Rockville, v. 118, n. 1, p. 363-373, July 2017.

ALLEN, Natalie et al. Bradykinesia, muscle weakness and reduced muscle power in Parkinson's disease. Movement Disorders, Madrid, v. 24, n. 9, p.1344-1351, July 2009.

ARCILA, Diana et al. Metodologia e didática pedagógica aplicada ao ensino da Caminhada Nórdica e Livre para pessoas com Doença de Parkinson. Cadernos de Formação RBCE, Porto Alegre, v. 8, n. 2, p. 72-83, set. 2017.

BARGHI, Effat; GLADDEN, Margaret. Motor unit number estimation in normal and parkinsonism model of medial gastrocnemius muscle in rats. International Journal of Molecular and Cellular Medicine, Babol, v. 2, n. 2, p. 72-79, 2013.

BRIENESSE, Laura; MICHAEL, Emerson. Effects of resistance training for people with Parkinson's disease: a systematic review. Journal of the American Medical Directors Association, New York, v. 14, n. 4, p. 236-241, Jan. 2013. 
BROWN, Portiau; CORCOS, Daniel; ROTHWELL, John. Does parkinsonian action tremor contribute to muscle weakness in Parkinson's disease? Brain, Oxford, v. 120, n. 3, p. 401-408, 1997.

CATALÁ, María; WOITALLA, Dirk; ARAMPATZIS, Adamantios. Central factors explain muscle weakness in young fallers with Parkinson's disease. Neurorehabilitation and Neural Repair, Thousand Oaks, v. 27, n. 8, p. 753-759, June 2013.

COLE, Michael; NAUGHTON, Geraldine; SILBURN, Peter. Neuromuscular impairments are associated with impaired head and trunk stability during gait in Parkinson fallers. Neurorehabilitation and Neural Repair, Thousand Oaks, v. 31, n. 1, p. 34-47, 2017.

COLLIER, Timothy; KANAAN, Nicholas; KORDOWER, Jeffrey. Aging and Parkinson's disease: different sides of the same coin? Movement Disorders, Madrid, v. 32, n. 7 , p. 983-990, July 2017.

DELABARY, Marcela et al. Effects of dance practice on functional mobility, motor symptoms and quality of life in people with Parkinson's disease: a systematic review with meta-analysis. Aging Clinical and Experimental Research, New York, v. 30, n. 7, p. 727-735, 2018.

DESCHENES, Michael. Effects of aging on muscle fibre type and size. Sports Medicine, New York, v. 34, n. 12, p. 809-824, 2004.

EBERSBACH, Georg et al. Comparing exercise in Parkinson's disease - the Berlin BIG Study. Movement Disorders, Madrid, v. 25, n. 12, p. 1902-1908, 2010.

FLORIANO, Eduardo Nascimento et al. Dual task performance: a comparison between healthy elderly individuals and those with Parkinson's disease. Fisioterapia em Movimento, Curitiba, v. 28, n. 2, p. 251-258, jun. 2015.

FOX, Cynthia et al. The science and practice of LSVT/LOUD: neural plasticity-principled approach to treating individuals with Parkinson's disease and other neurologica disorders. Seminars in Speech and Language, New York, v. 27, n. 4, p. 283-299, 2006

FRANZONI, Leandro et al. A 9-Week nordic and free walking improve postural balance in Parkinson's disease. Sports Medicine International Open, New York, v. 2, n. 1, p. 28-34, Nov. 2018

GOODWIN, Victoria et al. The effectiveness of exercise interventions for people with Parkinson's disease: a systematic review and meta-analysis. Movement Disorders, Singapore, v. 23, n. 5, p. 631-640, Jan. 2008.

GOUGEON, Marie-Anne; ZHOU, Lei; NANTEL, Julie. Nordic walking improves trunk stability and gait spatial-temporal characteristics in people with Parkinson disease. NeuroRehabilitation, Clifton, v. 42, n. 1, p. 205-210, 2017.

HAMMOND, Kelley et al. Neuromuscular rate of force development deficit in Parkinson disease. Clinical Biomechanics, London, v. 45, p. 14-18, 2017.

HEPPLE, Russell; RICE, Charles. Innervation and neuromuscular control in ageing skeletal muscle. The Journal of Physiology, Malden, v. 594, n. 8, p. 1965-1978, Dec. 2016

HUANG, Zhigao; FUENTE-FERNÁNDEZ, Raúl de La; STOESSL, Jon. Etiology of Parkinson's Disease. Canadian Journal Neurology Sciences, New York, v. 30, n. 1, p. 10-18, 2003

HUNTER, Sandra; PEREIRA, Hugo; KEENAN, Kevin. The aging neuromuscular system and motor performance. Journal of Applied Physiology, Rockville, v. 121, n. 4 p. 982-995, Ago. 2016.

INSTITUTO BRASILEIRO DE GEOGRAFIA E ESTATÍSTICA (IBGE). Indicadores sociais municipais: uma análise dos resultados do universo do Censo Demográfico. Rio de Janeiro: IBGE, 2009. Disponível em: https://ww2.ibge.gov.br/home/estatistica/popu lacao/censo2010/indicadores_sociais_municipais/default_indicadores_sociais_municipais.shtm. Acesso em: 25 set. 2017. 
JANKELOWITZ, Stacey; Burke, David. Do the motor Manifestations of Parkinson disease alter motor axon excitability? Muscle \& Nerve, Singapore, v. 45, n. 1, p. 43-47, 2012.

KWON, Young; YOON, Sung-Sang. Sarcopenia: Neurological Point of View. Journal of Bone Metabolism, Seoul, v. 24, n. 2, p. 83, mai. 2017.

LABAT-ROBERT, Jacqueline; ROBERT, Ladislas. Longevity and aging: mechanisms and perspectives. Pathologie Biologie, New Yokr, v. 63, n. 6, p. 272-276, Dec. 2015.

LAMOTTE, Guillaume et al. Effects of endurance exercise training on the motor and non-motor features of Parkinson's disease: a review. Journal of Parkinson's Disease, Netherlands, v. 5, n. 1, p. 21-41, 2015.

LEE, Han Suk; PARK, Jeung Hun. Effects of nordic walking on physical functions and depression in frail people aged 70 years and above. Journal of Physical Therapy Science, Netherlands, v. 27, n. 8, p. 2453-2456, 2015

MANINI, Todd; HONG, Lee; CLARK, Brian. Aging and muscle : a neuron's perspective. Current Opinion in Clinical Nutrition and Metabolic Care, London, v. 16, n. 1, Jan. 2013.

MCGILL, Ashley; HOUSTON, Sara; LEE, Raymond. Dance for Parkinson's: a new framework for research on its physical, mental, emotional, and social benefits. Complementary Therapies in Medicine, New York, v. 22, n. 3, p. 426-432, 2014.

MONTEIRO, Elren Passos et al. Aspectos biomecânicos da locomoção de pessoas com doença de Parkinson: revisão narrativa. Revista Brasileira de Ciências do Esporte, Porto Alegre, v. 39, n. 4, p. 450-457, ago. 2017.

MURRAY, Danielle et al. The effects of exercise on cognition in Parkinson's disease: a systematic review. Translational Neurodegeneration, New York, v. 3, n. 1, 2014.

PELLEGRINI, Barbara et al. Exploring muscle activation during nordic walking: a comparison between conventional and uphill walking. PloS One, San Francisco, v. 10, n. 9, 2015.

PELLEGRINI, Barbara et al. Mechanical energy patterns in nordic walking: comparisons with conventional walking. Gait \& Posture, New York, v. 51, p. 234-238, 2017.

PELLEGRINI, Barbara et al. Muscular and metabolic responses to different nordic walking techniques, when style matters. PloS One, San Francisco, v. 13, n. 4, 2018.

PERFEITO, Rita; REGO, Ana Carolina. Papel da alfa-sinucleína e da disfunção mitocondrial associada à doença de Parkinson. Revista Neurociencias, São Paulo, v. 20, n. 2, p. 273-284, out. 2012

PETERSON, Daniel; HORAK, Fay. Neural Control of Walking in People with Parkinsonism. Physiology, Rockville, v. 31, n. 2, p. 95-107, Mar. 2016.

PÍCOLI, Tatiane; FIGUEIREDO, Larissa; PATRIZZI, Lislei Jorge. Sarcopenia e envelhecimento. Fisioterapia em Movimento, Curitiba, v. 24, n. 3, p. 455-462, jul./set. 2011.

POLDRACK, Russell; PACKARD, Mark. Competition among multiple memory systems: converging evidence from animal and human brain studies. Neuropsychologia, New York, v. 41, n. 3, p. 245-251, 2003.

RODRIGUEZ, Kathryn et al. Persons with Parkinson's disease exhibit decreased neuromuscular complexity during gait. Clinical Neurophysiology, New York, v. 124, n. 7, p. 1390-1397, Mar. 2013.

RODRIGUEZ, Manuel et al. Parkinson's disease as a result of aging. Aging Cell, London, v. 14, n. 3, p. 293-308, Nov. 2015.

SCHNEIDER, Susanne; ALCALAY, Roy. Neuropathology of genetic synucleinopathies with parkinsonism: review of the literature. Movement Disorders, Madrid, v. 32, n. 11, p. 1504-1523, Sep. 2017. 
SHANAHAN, Joanne et al. Dance for people with Parkinson disease: what is the evidence telling us? Archives of Physical Medicine and Rehabilitation, New York, v. 96, n. 1, p. 141-153, 2015.

SHARP, Kathryn; HEWITT, Jonathan. Dance as an intervention for people with Parkinson's disease: a systematic review and meta-analysis. Neuroscience and Biobehavioral Reviews, New York, v. 47, p. 445-456, Sep. 2014

SHU, Hai-Feng et al. Aerobic exercise for Parkinson's disease: a systematic review and meta-analysis of randomized controlled trials. PloS One, San Francisco, v. 9, n. 7, July 2014.

SKÓRKOWSKA-TELICHOWSKA, Katarzyna et al. Nordic walking in the second half of life. Aging Clinical and Experimental Research, New York, v. 28, n. 6, p. 1035-1046, 2016.

THAUT, M. H. et al. The connection between rhythmicity and brain function: implications for therapy of movement disorders. IEEE Engineering in Medicine and Biology Magazine, [s. I.], v. 18, n. 2, p. 101-108, 1999.

TSCHENTSCHER, Marcus; NIEDERSEER, David; NIEBAUER, Josef. Health benefits of nordic walking: a systematic review. American Journal of Preventive Medicine, Ann Arbor, v. 44, n. 1, p. 76-84, 2013.

TUDORAŞCU, lulia et al. Motor unit changes in normal aging: a brief review. Romanian Journal of Morphology and Embryology, Craiova, v. 55, n. 4, p. 1295-1301, 2014.

VAN EIJKEREN, Frank et al. Nordic walking improves mobility in Parkinson's disease. Movement Disorders, Madrid, v. 23, n. 15, p. 2239-2243, 2008.

WILD, Lucia et al. Characterization of cognitive and motor performance during dual-tasking in healthy older adults and patients with Parkinson's disease. Journal of Neurology, New York, v. 260, n. 2, p. 580-589, 2013.

WORLD HEALTH ORGANIZATION (WHO). Proposed working definition of an older person in Africa for the MDS Project. Geneva: WHO, 2009. Disponivel em: http://www. who.int/healthinfo/survey/ageingdefnolder/en. Acesso em: 25 set. 2017.

WU, Rui et al. Effects of age and sex on neuromuscular-mechanical determinants of muscle strength. Journal of the American Aging Association, Grandville, v. 38, n. 3, 2016.

Data de Submissão: 26/10/2018

Data de Aprovação: 30/11/2018 
